www.jmscr.igmpublication.org

Impact Factor 5.244

Index Copernicus Value: 5.88

ISSN (e)-2347-176x ISSN (p) 2455-0450

crossref DOI:_http://dx.doi.org/10.18535/jmscr/v4i5.45

Journal Of Medical Science And Clinical Research

\title{
Stress Induced Cardiomyopathy Is Rare -Case Report
}

\author{
Author \\ Dr Gopalakrishnan.M
}

MD, DCARD, FICC, Consultant Cardiologist, Holy Family Hospital, Thodupuzha, Kerala

\begin{tabular}{l}
\hline ABSTRACT \\
Stress Induced Cardiopyopathy Is Rare Disorder, But Increasing Incidence Among Emotional Sterss, \\
Anxiety, Post Menopausal Women. \\
\hline
\end{tabular}

\section{INTRODUCTION}

Stress induced cardiomyopathy is a very rare condition and has to be differentiated from myocardial infarct. The incidence of Stress induced cardiomyopathy is $1-2 \%$ in patients diagnosed with MI. These individuals are usually postmenopausal females (90\%). The average age is 62-75 years old.

Usually Stress induced cadiomyopathy is triggered by emotional stress, physical stress. The onset is insidious and presents with chest pain at rest. Must have all 4 criteria to be diagnosed

- Transient hypokinesis, akinesis, or dyskinesis of the LV midsegments

- Absence of obstructive coronary artery or angiographic evidence of acute plaque rupture

- New ECG abnormalities or elevated cardiac troponin

- Absence of recent head trauma, intracranial bleeding, pheochromocytoma, myocarditis, and hypertrophic cardiomyopathy

\section{CASE REPORT}

A 65 year old post menopausal lady, known $\mathrm{dm}, \mathrm{hth}$ on regular medications came with $\mathrm{h} / \mathrm{o}$ anterior chest discomfort radiating to back around 12 hours duration on and off . no h/o doe, no h/o aoe past .she is a administrator by occupation .on examination cvs - s1 s2 +, no murmur ,rs- vbs ,no added sounds, abdomen -soft , non tender,no organomegaly

ECG- sr, poor progression of $r$ wave in $v 1-v 4$. echo - mid, distal ivs apex hypokinetic, basal ivs,posterior wall hypercontractile, mild $\mathrm{lv}$ systolic dysfunction, lvef- $50 \%$.

troponin $-\mathrm{i} \quad 0.06 \mathrm{ug} / \mathrm{l}$ (normal less then $0.01 \mathrm{ug} / \mathrm{l}$ ) hb 13.6 gm\%,wbc 9500 ul,platelet count 181000 lakhs/ul,urea $25 \mathrm{mg}$, creatinine $0.7 \mathrm{mg}$, na 135 $\mathrm{meq} / \mathrm{l}, \mathrm{k} 4 \mathrm{~m} \mathrm{eq} / \mathrm{l}$, cl $105 \mathrm{~m}$ eq/l.urine routine normal ,total cholestrol $204 \mathrm{mg}$,tgl $243 \mathrm{mg}$,hdl $37 \mathrm{mg}$,ldl $117 \mathrm{mg}$, rbs 120mg.

Cag -insignificant cad (prox lad minor plaque,major om $30 \%$ ).

Patient was diagnosed to have sterss induced cardiomyopathy. 
Patient is managed with t.ecosprin, t.clopilet, t.atorva, t.telmisartan, oha other supportive medications. Patient is given for complete bed rest.

After 3 days echocardiogram repeated, which was shown no rwma, normal lv systolic function, lvef$60 \%$.

\section{CONCLUSION}

Sterss Induced Cardiomyopathy Is Rare, And It's A Non Ischemic Cardiomyopathy, Increasing Incidence Among Postmenopausal Women.

\section{REFERENCES}

1. Gianni M, Dentali F, et al. (December 2006). "Apical ballooning syndrome or takotsubo cardiomyopathy: a systematic review". European Heart Journal (Oxford University Press) 27 (13): 1523-1529.

2. Azzarelli S, Galassi AR, Amico F, Giacoppo M, Argentino V, Tomasello SD, Tamburino C, Fiscella A. (2006). "Clinical features of transient left ventricular apical ballooning". Am J Cardiol. 98 (9): 1273-6.

3. Kurisu S, Sato $H$, Kawagoe $T$, et al. (2002). "Tako-tsubo-like left ventricular dysfunction with ST-segment elevation: a novel cardiac syndrome mimicking acute myocardial infarction". American Heart Journal 143 (3): 448-55.

4. Schneider B., Athanasiadis A., Sechtem U. (2013). "Gender-Related differences in Takotsubo Cardiomyopathy". Heart Failure Clinics 9 (2): 137-146. 5.Pilgrim TM, Wyss TR (March 2008). "Takotsubo cardiomyopathy or transient left ventricular apical ballooning syndrome: A systematic review". Int. J. Cardiol. 124 (3): 283-92.

5. Kumar G, Holmes DR Jr, Prasad A. "Familial" apical ballooning syndrome (Takotsubo cardiomyopathy). Int J Cardiol 2010; $144: 444$. 